\title{
On random symmetric travelling salesman problems
}

\author{
Alan Frieze \\ Department of Mathematical Sciences, \\ Carnegie Mellon University. \\ alan@random.math.cmu.edu*
}

July 8,2002

\begin{abstract}
Let the edges of the complete graph $K_{n}$ be assigned independent uniform [0,1] random edge weights. Let $Z_{T S P}$ and $Z_{2 F A C}$ be the weights of the minimum length travelling salesman tour and minimum weight 2 -factor respectively. We show that $\mathbf{w h p}^{1}$ $\left|Z_{T S P}-Z_{2 F A C}\right|=o(1)$. The proof is via by the analysis of a polynomial time algorithm that finds a tour only a little longer than $Z_{2 F A C}$.
\end{abstract}

\section{Introduction}

The starting point of this line of research is the foundational paper of Karp [9] in 1979. Karp considered the following problem: The arcs of the complete digraph $D_{n}$ on vertex set $[n]$ are given independent uniform $[0,1]$ random edge weights. $Z_{T S P}$ denotes the weight of the minimum length (directed) travelling salesman tour and $Z_{A S S}$ denotes the minimum weight of an assignment for the associated $n \times n$ matrix $\mathbf{M}$ of costs. Karp proved, via the analysis of an $O\left(n^{3}\right)$ time algorithm, that whp $\left|Z_{T S P}-Z_{A S S}\right|=o(1)$. This gave good theoretical backing for the empirical observation (see e.g. Balas and Toth [2]) that the assignment problem provides a good lower bound for use in branch and bound algorithms. A sequence of papers, Karp and Steele [10], Dyer and Frieze [4] and Frieze and Sorkin [8] tightened this result. In particular, [8] proves that

$$
\left|Z_{T S P}-Z_{A S S}\right|=O\left(\frac{(\ln n)^{2}}{n}\right) \quad \text { whp. }
$$

It is natural to try to prove a similar result for symmetric problems. Here the edges of the complete graph $K_{n}$ are assigned independent uniform $[0,1]$ random edge weights. Up to now there has been almost no progress analysing this probabilistic model of the TSP. Let $\mathbf{M}=\mathbf{M}(i, j)$ once again denote the matrix of lengths. Here of course $\mathbf{M}$ is symmetric i.e. $\mathbf{M}(i, j)=\mathbf{M}(j, i)$ for all $i, j \in[n]$. Let $Z_{T S P}=Z_{T S P}(\mathbf{M})$ denote the length of the shortest Hamilton cycle. It is unlikely that solving the assignment problem for $\mathbf{M}$ will yield a good approximation to $Z_{T S P}$ since its solution $(i, \pi(i)), i \in[n]$ will likely contain many instances $i, j$ where $\pi(i)=j$ and $\pi(j)=i .^{2}$ It seems more sensible to start with the solution of the minimum weight 2factor problem. A 2-factor is a subgraph of $K_{n}$ in which every vertex has degree 2 and so is a

\footnotetext{
${ }^{*}$ Supported in part by NSF grant CCR-9818411.

${ }^{1}$ By "with high probability" (whp) we mean "with probability 1-o(1) as $n \rightarrow \infty$ "

${ }^{2}$ Perhaps one could try to prove that $Z_{2 F A C}-Z_{A S S}=\Omega(1)$ whp.
} 
collection of vertex disjoint cyles which cover all vertices in $[n]$. A minimum weight 2-factor can be constructed in polynomial time. It is a classical problem in matching theory, see e.g. Lovász and Plummer [11]. Let $Z_{2 F A C}=Z_{2 F A C}(\mathbf{M})$ be the weight of the minimum weight 2-factor. A tour i.e. a cycle with $n$ edges is a 2 -factor and so clearly $z_{2 F A C} \leq z_{T S P}$. Our main result is:

\section{Theorem 1}

$$
z_{T S P}-z_{2 F A C}=o(1) \quad \text { whp. }
$$

Furthermore, whp a tour of length $z_{2 F A C}+o(1)$ can be constructed by a polynomial time algorithm.

Now $z_{T S P} \geq z_{M S T}$, the weight of the minimum spanning tree, and whp $z_{M S T} \geq \zeta(3)-o(1) \sim$ $1.202 \ldots$, Frieze [6]. Thus the tour produced by our algorithm is asymptotically optimal.

It as well to point out now what makes the symmetric case seemingly more difficult than the asymmetric case studied in [9], [10], [4] and [8]. The solution to the assignment problem can be represented as a permutation $\left(i, \pi^{*}(i)\right), i \in[n]$. It is straightforward to show that the distribution of $\pi^{*}$ is uniform over the set of possible permutations. As such, the number of cycles of $\pi^{*}$ is $O(\ln n)$ whp. This is a great help in the analysis. Now a random 2-factor will also have $O(\ln n)$ cycles whp but it is not at all clear that the minimum weight 2 -factor has a uniform distribution.

If it did, then we could replace the o(1) in (1) by $O\left(\frac{(\ln n)^{2}}{n}\right)$. Thus one of our problems has been to put a high probability bound on the number of cycles in the minimum weight 2 -factor. We have not been too successful. We have only managed a "miserable" $O\left(\frac{n}{\ln n}\right)$ which is just on the borderline of being useful. Any fewer and the paper would be much shorter. We will however have to content ourselves with what we have and make the best of it.

Structure of the paper: In the next section we prove some preliminary results about the structure of the minimum weight 2-factor $F^{*}=F^{*}(\mathbf{M})$. In Section 3 we will describe a model for use given the cycle structure of $F^{*}$. In Section 4 we will describe our algorithm for finding a tour and prove that it is asymptotically optimal.

\section{Preliminary Analysis}

\section{$2.1 \quad$ Upper bound on $Z_{T S P}$}

The first thing we shall do is prove a high probability upper bound on $z_{T S P}$. It is quite weak and obtaining a more precise bound remains an interesting open problem. (The work of Aldous [1] combined with Karp [9] shows that for the asymmetric case, $z_{T S P} \sim \frac{\pi^{2}}{6} \mathbf{w h p}$ ).

\section{Lemma 1}

$$
z_{T S P} \leq 6 \quad \text { whp. }
$$

Proof We use an old idea of Walkup [12]. Let $Z$ be a random variable on $[0,1]$ with $\operatorname{Pr}(Z \leq x)=1-\sqrt{1-x}$ for $x \in[0,1]$. Then if $Z_{1}, Z_{2}$ are independent copies of $Z, \min \left\{Z_{1}, Z_{2}\right\}$ is distributed as a uniform $[0,1]$ random variable. So now for $i<j$ let the edge lengths $\mathbf{M}(i, j)$ be generated by $\min \left\{Z_{1}(i, j), Z_{1}(j, i)\right\}$ where the set $Z_{1}(i, j), 1 \leq i \neq j \leq n$ are independent copies of $Z$.

For each integer $m \geq 1$ define a random digraph $\vec{\Gamma}_{m}$ with vertex set $[n]$ and a directed arc $(x, y)$ if $Z_{1}(x, y)$ is one of the $m$ smallest values $Z_{1}(x, j), j \neq x$. By ignoring orientation in $\vec{\Gamma}_{m}$ we obtain 
the random graph $\Gamma_{m}=G_{m-o u t}$. Cooper and Frieze [3] showed that $\Gamma_{4}$ is Hamiltonian whp. If $H$ is a randomly chosen Hamilton cycle of $\Gamma_{4}$ then each edge has expected length $\leq \frac{2+4+6+8}{4 n}$ and so the expected length of $H$ is $\leq 5$. It will be $\leq 6 \mathrm{whp}$ and its length bounds $z_{T S P}$ from above.

\subsection{The number of cycles in $F^{*}$}

We can use Lemma 1 to help bound the number of cycles in a minimum weight 2-factor.

Lemma 2 Whp $F^{*}$ consists of at most $\frac{3 n}{\ln n}$ cycles.

Proof Let $Z$ denote the number of cycles in the minimum weight 2 -factor. For $3 n / \ln n \leq$ $r \leq n / 3$, we bound the probability that $Z=r$ by the expected number of 2 -factors with $r$ cycles and total weight $\leq 6$. The o(1) additive term below is from Lemma 1 .

$$
\begin{aligned}
\operatorname{Pr}(Z \geq r) & \leq o(1)+\sum_{r=3 n / \ln n}^{n / 3} \sum_{k_{1}+\cdots+k_{r}=n} \frac{1}{r !}\left(\begin{array}{c}
n ! \\
k_{1}, \ldots, k_{r}
\end{array}\right) \prod_{i=1}^{r} \frac{\left(k_{i}-1\right) !}{2} \frac{6^{n}}{n !} \\
& =o(1)+\sum_{r=3 n / \ln n}^{n / 3} \frac{6^{n}}{2^{r} r !} \sum_{k_{1}+\cdots+k_{r}=n} \prod_{i=1}^{r} \frac{1}{k_{i}} \\
& =o(1)+\sum_{r=3 n / \ln n}^{n / 3} \frac{6^{n}}{2^{r} r !}\left[x^{n}\right](-\ln (1-x))^{r} \\
& \leq o(1)+\sum_{r=3 n / \ln n}^{n / 3} \frac{6^{n}}{2^{r} r !} \frac{(-\ln (1-x))^{r}}{x^{n}} \quad \forall 0<x<1 \\
& =o(1)+\sum_{r=3 n / \ln n}^{n / 3} \frac{(12)^{n}(\ln 2)^{r}}{2^{r} r !} \\
& \leq o(1)+\sum_{r=3 n / \ln n}^{n / 3}(12)^{n}\left(\frac{e}{2 r}\right)^{r} \\
& \leq o(1)+\sum_{r=3 n / \ln n}^{n / 3} e^{-n / 2} \\
& =o(1) . \quad{ }^{n} \quad{ }^{n}
\end{aligned}
$$

\subsection{The longest edge in $F^{*}$}

Let $\Gamma_{20000}$ be as defined in the proof of Lemma 1. We first show that whp $\Gamma_{20000}$ is a good expander. For $S \subseteq[n]$ let

$$
\begin{aligned}
& \vec{N}_{20000}(S)=\left\{w \notin S: \exists v \in S \text { such that }(v, w) \text { is an arc of } \vec{\Gamma}_{20000}\right\} . \\
& N_{20000}(S)=\left\{w \notin S: \exists v \in S \text { such that }(v, w) \text { is an edge of } \Gamma_{20000}\right\} .
\end{aligned}
$$

Lemma 3 Whp $\left|\vec{N}_{20000}(S)\right| \geq 20|S|$ for all $S \subseteq[n],|S| \leq n / 25$. 
Proof

$$
\begin{aligned}
\operatorname{Pr}\left(\exists S:|S| \leq\lceil n / 25\rceil,\left|\vec{N}_{20000}(S)\right|<20|S|\right) & \left.\leq \sum_{s=1}^{\lceil n / 25\rceil}\left(\begin{array}{c}
n \\
s
\end{array}\right)\left(\begin{array}{c}
n \\
20 s
\end{array}\right)\left(\frac{\left(\begin{array}{c}
21 s \\
20000 \\
n
\end{array}\right)}{(20000}\right)\right)^{s} \\
& \leq \sum_{s=1}^{\lceil n / 25\rceil}\left(\frac{n e}{s}\right)^{s}\left(\frac{n e}{20 s}\right)^{20 s}\left(\frac{21 s}{n}\right)^{20000 s} \\
& =\sum_{s=1}^{\lceil n / 25\rceil}\left(\frac{e^{21}(21)^{20000} s^{19979}}{(20)^{20} n^{19979}}\right)^{s} \\
& =o(1) .
\end{aligned}
$$

Every pair of large subsets of $\Gamma_{20000}$ are joined by many edges:

Lemma 4 Whp $\Gamma_{20000}$ contains at least $2 n$ edges joining $S$ and $T$, for all $S, T \subseteq[n],|S|,|T| \geq$ $n / 50$ and $S \cap T=\emptyset$.

Proof For fixed disjoint sets $S, T$ of size $n / 50$, the number of edges $X$ joining $S$ and $T$ dominates the binomial $B\left(800 n, \frac{1}{50}\right)$. Thus, by Chernoff bounds,

$$
\operatorname{Pr}(X \leq 3 n) \leq e^{-5 n}
$$

So the probability there exists a pair $S, T$ for which $X \leq 3 n$ is at most

$$
\left(\begin{array}{c}
n \\
n / 50
\end{array}\right)^{2} e^{-5 n}=o(1)
$$

(We should take account of multiple edges here, but whp there are $o(n)$ of these).

No two vertices have many common neighbours in $\Gamma_{20000}$.

Lemma 5 Whp every pair of vertices $x, y$ have at most 2 common neighbours in $\Gamma_{20000}$.

Proof The probability that there are a pair of vertices $x, y$ with 3 common neighbours in $\Gamma_{20000}$ is at most

$$
\left(\begin{array}{l}
n \\
2
\end{array}\right)\left(\begin{array}{l}
n \\
3
\end{array}\right)\left(\frac{40000}{n}\right)^{6}=o(1)
$$

since the probability that a pair of vertices are adjacent in $\Gamma_{20000}$ is at most $\frac{40000}{n}$ and this probability is reduced if we are given the existence of other edges.

Now let $F$ be an arbitrary 2-factor. We consider alternating paths in $\Gamma_{20000}$ with respect to $F$ i.e. paths of the form $x_{0}, x_{1}, \ldots, x_{k}$ where the edges $\left(x_{2 i}, x_{2 i+1}\right) \notin F$ for $0 \leq i \leq\lceil k / 2\rceil$ and the edges $\left(x_{2 i-1}, x_{2 i}\right) \in F$ for $1 \leq i \leq\lfloor k / 2\rfloor$.

Lemma 6 Whp for every 2-factor $F$ and for every pair of vertices $x, y$ there is an odd length alternating path from $x$ to $y$ of length at most $2 i_{0}+1$ where $i_{0}=1+\left\lceil\log _{2}(n /(50 \times 19996)\rceil\right.$.

Proof Assume that the conditions of Lemmas 3, 4 and 5 hold.

Fix $x, y, F$ and arbitrarily orient the edges of $F$ to obtain $\vec{F}$. For a vertex $z$ let $\nu(z)$ be defined by $(z, \nu(z))$ is an arc of $\vec{F}$.

We define a collection of sets $S_{0}=\{x\}, T_{0}=\{y\}, S_{i}, T_{i}, i=1,2, \ldots, i_{0}$ where 
(i) The sets $S_{i}, \nu\left(S_{i}\right), T_{i}, \nu\left(T_{i}\right), i=1,2, \ldots, i_{0}$ are pair-wise disjoint.

(ii) $S_{i}$ is reachable from $x$ by an alternating path of length $2 i-1$ (number of edges), $1 \leq i \leq i_{0}$.

(iii) $T_{i}$ is reachable from $y$ by an alternating path of length $2 i-1,1 \leq i \leq i_{0}$.

(iv) $\left|S_{1}\right|=\left|T_{1}\right|=19996$.

(v) $\left|S_{i+1}\right|=2\left|S_{i}\right|$ and $\left|T_{i+1}\right|=2\left|T_{i}\right|$ for $1 \leq i<i_{0}$.

Since (Lemma 5) $\left|N_{20000}(x) \cap N_{20000}(y)\right| \leq 2$ we can choose $\tilde{S}_{1} \subseteq N_{20000}(x), \tilde{T}_{1} \subseteq N_{20000}(y)$ such that $\left|\tilde{S}_{1}\right|=\left|\tilde{T}_{1}\right|=19998$ and $\tilde{S}_{1}, \tilde{T}_{1}$ are disjoint. At most 2 of the $x, \tilde{S}_{1}$ edges are in $F$ and so we can choose 19996 members of $\tilde{S}_{1}$ to make $S_{1}$ and similarly we can choose 19996 members of $\tilde{T}_{1}$ to make $T_{1}$.

Now suppose that we have chosen $S_{i}, T_{i}$ for some $1 \leq i<i_{0}$ and that $s=\left|S_{i}\right|=\left|T_{i}\right|$. Let $A_{0}=N_{20000}\left(S_{i}\right), B_{0}=N_{20000}\left(T_{i}\right)$ and $C=N_{20000}\left(S_{i} \cup T_{i}\right) \subseteq A \cup B$. Since $\left|A_{0}\right|,\left|B_{0}\right| \geq 20 s$ and $|C| \geq 40 \mathrm{~s}$ we can choose $A_{1} \subseteq A_{0}, B_{1} \subseteq B_{0}$ such that $A_{1} \cap B_{1}=\emptyset$ and $\left|A_{1}\right|=\left|B_{1}\right|=20 \mathrm{~s}$. By deleting $2 s$ vertices from $A_{1}$ we obtain a set $A_{2} \subseteq A_{0}$ of size $18 s$ such that all $S_{i}: A_{2}$ edges are not in $F$. Define $B_{2}$ analogously.

Next let $A_{3}=\nu\left(A_{2}\right)$ and $B_{3}=\nu\left(B_{2}\right)$. At this point the only possible intersections among $A_{2}, A_{3}, B_{2}, B_{3}$ are between $A_{3}$ and $B_{2}$ or between $A_{2}$ and $B_{3}$. Now choose $6 s$ vertices $A_{4} \subseteq A_{3}$ and let $A_{5}=\nu^{-1}\left(A_{4}\right)$. Next choose $6 s$ vertices $B_{4}$ from $B_{3} \backslash\left(\nu\left(A_{4}\right) \cup A_{5}\right)$. By so doing we have $A_{4}, A_{5}, B_{4}, B_{5}=\nu^{-1}\left(B_{4}\right)$ disjoint.

Since

$$
\left|\bigcup_{j=0}^{i-1}\left(S_{j} \cup \nu^{-1}\left(S_{j}\right) \cup T_{j} \cup \nu^{-1}\left(T_{j}\right)\right)\right| \leq 4 s
$$

we can find $S_{i+1} \subseteq A_{4}, T_{i+1} \subseteq B_{4}$ so that (i) above holds and so complete the inductive step.

Now $\left|S_{i_{0}}\right|,\left|T_{i_{0}}\right| \geq n / 50$ and so (Lemma 4 ) there are at least $2 n$ edges joining these 2 sets, at least one of which is not in $F$. This proves the existence of the required alternating path.

We now define the weight of an alternating path $P$ w.r.t. $F^{*}$ to be the difference between the sum of the weights of the edges of $P$ which are not in $F^{*}$ and the sum of the weights of the edges of $P$ which are in $F^{*}$.

Lemma 7 There exists an absolute constant $C_{1}>0$ such that whp every pair of vertices is joined by an alternating path of weight $\leq \frac{C_{1} \ln n}{n}$.

Proof We will need the following inequality, Lemma 4.2(b) of Frieze and Grimmett [7].

Suppose that $k_{1}+k_{2}+\cdots+k_{s} \leq a$, and $Y_{1}, Y_{2}, \ldots, Y_{s}$ are independent random variables with $Y_{i}$ distributed as the $k_{i}$ th minimum of $N$ independent uniform $[0,1]$ random variables. If $\mu>1$ then

$$
\operatorname{Pr}\left(Y_{1}+\cdots+Y_{s} \geq \frac{\mu a}{N+1}\right) \leq e^{a(1+\ln \mu-\mu)}
$$

Let

$$
Z_{1}=\max \left\{\sum_{i=0}^{k} \mathbf{M}\left(x_{2 i}, x_{2 i+1}\right)-\sum_{i=1}^{k} \mathbf{M}\left(x_{2-1}, x_{2 i}\right)\right\}
$$


where the maximum is over sequences $x_{0}, x_{1}, \ldots, x_{2 k+1}$ where $k \leq i_{0}$ and $\mathbf{M}\left(x_{2 i}, x_{2 i+1}\right), i=$ $0,1, \ldots, k$ is stochastically dominated by the 20000th smallest of $n-3$ independent copies of the random variable $Z$ of Lemma 1 . (Here we can use the fact that in our definition of an alternating path the weight of $\left(x_{2 i}, x_{2 i+1}\right)$ is stochastically dominated by the minimum of the $n-3 Z_{1}\left(x_{2 i}, y\right)$ 's corresponding to edges not in $F$. Notice that this quantity is independent of the length of $\left.\left(x_{2 i+1}, x_{2 i+2}\right)\right)$. The remaining edge lengths are uniform and all lengths can be taken as independent. Because $1-\sqrt{1-x} \geq x / 2$ for $x \in[0,1]$ we see that $Z$ is stochastically dominated by $2 U$ where $U$ is uniform $[0,1]$.

We estimate the probability that $Z_{1}$ is large. Indeed, for any $\zeta>0$ we have

$$
\begin{aligned}
\operatorname{Pr}\left(Z_{1} \geq \zeta \frac{\ln n}{n}\right) \leq \sum_{k=1}^{i_{0}} n^{2 k+2} & \frac{1}{(n-3)^{k+1}} \frac{1}{k !} \times \\
& \int_{y=0}^{\infty}\left[\left(\frac{y \ln n}{n}\right)^{k} \sum_{\rho_{1}+\cdots+\rho_{k+1} \leq 20000(k+1)} q\left(\rho_{1}, \ldots, \rho_{k+1} ; \zeta+y\right)\right] d y
\end{aligned}
$$

where

$$
q\left(\rho_{1}, \ldots, \rho_{k+1} ; \eta\right)=\operatorname{Pr}\left(X_{1}+\cdots+X_{k+1} \geq \eta \frac{\ln n}{n}\right)
$$

$X_{1}, \ldots, X_{k+1}$ are independent and $X_{j}$ is distributed as twice the $\rho_{j}$ th minimum of $n-3$ independent copies of $Z$.

Explanation: We have at most $n^{2 k+2}$ choices for the sequence $x_{0}, x_{1}, \ldots, x_{2 k+1}$. The term $\frac{1}{k !}\left(\frac{y \ln n}{n}\right)^{k}$ bounds the probability that the sum of $k$ independent uniforms, $\mathbf{M}\left(x_{1}, x_{2}\right)+\cdots+$ $\mathbf{M}\left(x_{2 k-1}, x_{2 k}\right)$, is at most $\frac{y \ln n}{n}$. We integrate over $y \cdot \frac{1}{n-3}$ is the probability that $\left(x_{2 i}, x_{2 i+1}\right)$ is the $\rho_{i}$ th shortest (in terms of $Z_{1}$ ) edge leaving $x_{2 i}$, and these events are independent for $0 \leq i \leq k-1$. The final summation bounds the probability that the associated edge lengths sum to at least $\frac{(\zeta+y) \ln n}{n}$.

It follows from (2) that if $\zeta$ is sufficiently large then, for all $y \geq 0, q\left(\rho_{1}, \ldots, \rho_{k} ; \zeta+y\right) \leq n^{-(\zeta+y) / 4}$ and

$$
\begin{aligned}
\operatorname{Pr}\left(Z_{1} \geq \zeta \frac{\ln n}{n}\right) & \leq 2 n^{1-\zeta / 4} \sum_{k=1}^{i_{0}} \frac{(\ln n)^{k}}{k !}\left(\begin{array}{c}
20000(k+1)-1 \\
k
\end{array}\right) \int_{y=0}^{\infty} y^{k} n^{-y / 4} d y \\
& \leq 2 n^{1-\zeta / 4} \sum_{k=1}^{i_{0}} \frac{(\ln n)^{k}}{k !}\left(\frac{160000 e}{\ln n}\right)^{k+1} \Gamma(k+1) \\
& \leq 2 n^{1-\zeta / 4}(160000 e)^{i_{0}+2} \\
& =o(1) .
\end{aligned}
$$

for sufficiently large $\zeta$.

The following lemma is almost immediate:

Lemma 8 Whp $F^{*}$ contains no edge longer than $C_{1} \frac{\ln n}{n}$.

Proof Suppose that $F^{*}$ contains an edge $e=(x, y)$ of length greater than $C_{1} \frac{\ln n}{n}$. Construct the alternating $P$ path from $x$ to $y$ promised by Lemma 7. By removing $e$ and the $F^{*}$ edges of $P$ from $F^{*}$ and replacing them with the non- $F^{*}$ edges of $P$ we obtain a 2 -factor of lower weight. 
Note that $\operatorname{whp} F^{*}$ contains an edge of length $\geq \frac{\ln n}{n}$. The distribution of the subgraph induced by edges of length $\leq p$ is the random graph $G_{n, p}$ for any $p \in[0,1]$ and we need $p \geq \frac{\ln n}{n}$ in order that $\delta\left(G_{n, p}\right) \geq 2$ whp.

We use the notation $p_{0}=C_{1} \frac{\ln n}{n}$ for the remainder of the paper.

As consequence of Lemma 8 , we see that whp $F^{*}$ does not contain many very short cycles.

Lemma 9 Whp $F^{*}$ contains at most $n^{3 / 4}$ cycles with fewer than $\frac{\ln n}{2 \ln \ln n}$ edges.

Proof Let $X_{k}$ denote the number of cycles of $K_{n}$, with $k$ or fewer edges, all of whose edges are of length at most $p_{0}$. Then

$$
\mathbf{E}\left(Z_{k}\right)=\sum_{l=3}^{k}\left(\begin{array}{l}
n \\
l
\end{array}\right) \frac{(l-1) !}{2} p_{0}^{l} \leq \sum_{l=3}^{k} \frac{\left(C_{1} \ln n\right)^{l}}{2 l} \leq\left(C_{1} \ln n\right)^{k} \leq n^{1 / 2+o(1)}
$$

if $k \leq \frac{\ln n}{2 \ln \ln n}$. Now use the Markov inequality.

Incidentally, this is one place where trying to use the assignment bound $Z_{A S S}$ would cause trouble. There is no obvious limit to the number of double edges in the optimum assignment.

\subsection{Long and short edges}

We can divide the edges of $K_{n}$ into long, length $\geq p_{0}$ and short edges. From the previous section we see that whp it is enough to find a minimum weight 2-factor in the graph induced by the short edges. If for each short edge $(x, y)$ we generate an extra parallel edge with length uniform in the range $\left[p_{0}, 1\right]$ then we can consider that we start with $G_{n, p_{0}}$, with edge weights uniform in $\left[0, p_{0}\right]$ plus an independent $K_{n}$ with edge weights uniform in $\left[p_{0}, 1\right]$ where we always use the shortest edge between a pair of vertices $x, y$.

We further divide the long edges into very long, length $\geq 2 p_{0}$ and medium length edges. Thus we will obtain another Red copy of $G_{n, p_{0}}$ with weights in the range $\left[p_{0}, 2 p_{0}\right]$ and a Blue copy of $K_{n}$ with edge lengths in the range $\left[2 p_{0}, 1\right]$.

It is important to realise that when we say we use an edge of a particular graph, say a red edge, we are really just upper bounding the length of the edge in the original $K_{n}$.

\section{A Conditional Model}

For a permutation $\pi$ of $[n]$ and matrix of weights $\mathbf{M}$ we define $\mathbf{M}_{\pi}$ by $\mathbf{M}_{\pi}(i, j)=\mathbf{M}(\pi(i), \pi(j))$. Clearly $\mathbf{M}$ and $\mathbf{M}_{\pi}$ have the same distribution. So for any 2 -factor $F$,

$$
\operatorname{Pr}\left(F^{*}(\mathbf{M})=F\right)=\operatorname{Pr}\left(F^{*}\left(\mathbf{M}_{\pi}\right)=F .\right.
$$

But $F^{*}\left(\mathbf{M}_{\pi}\right)=F$ iff $F^{*}(\mathbf{M})=F \circ \pi$ where $(i, j)$ is an edge of $F \circ \pi$ iff $(\pi(i), \pi(j))$ is an edge of $F$. So

$$
\operatorname{Pr}\left(F^{*}=F\right)=\operatorname{Pr}\left(F^{*}=F \circ \pi\right) .
$$

Now as $\pi$ ranges over the $n$ ! permutations of $[n], F \circ \pi$ ranges over all 2-factors having the same cycle structure as $F$ - cycle $i_{1}, i_{2}, \ldots, i_{l}, i_{1}$ of $F$ is mapped to cycle $\pi^{-1}\left(i_{1}\right), \pi^{-1}\left(i_{2}\right), \ldots, \pi^{-1}\left(i_{l}\right)$, $\pi^{-1}\left(i_{1}\right)$ of $F \circ \pi$. By symmetry each of these 2-factors appears the same number of times. 
For $\mathbf{k}=3 \leq k_{1} \leq k_{2} \leq \cdots \leq k_{m}$ we let $\Omega_{\mathbf{k}}$ denote the set of 2 -factors with these cycle sizes. If we compute $F^{*}$ by first choosing a random permutation $\pi$, then computing $F^{*}\left(\mathbf{M}_{\pi}\right)$ and then taking $F^{*}\left(\mathbf{M}_{\pi}\right) \circ \pi$ then we see that:

$$
\text { Given } F^{*} \in \Omega_{\mathbf{k}}, F^{*} \text { is a random member of } \Omega_{\mathbf{k}} \text {. }
$$

So we will now fix the cycle sizes $\mathbf{k}$ and assume that the conditions of Lemmas 2,9 hold. We will run our proposed algorithm under the assumption that we know $\mathbf{A}=\mathbf{M}_{\pi}$ and $\tilde{F}=F^{*}\left(\mathbf{M}_{\pi}\right)$ but that $\pi$ is a random permutation that we will expose as necessary. More precisely we assume that

(i) $F^{*}(\mathbf{A}) \in \Omega_{\mathbf{k}}$.

(ii) The conditions of Lemma 10 below are satisfied.

(iii) We have the graphs $H_{i}, i=1,2, \ldots, \lambda_{1}$ of Section 2.4 .

It will help to imagine that we have $m$ cycles $C_{1}, C_{2}, \ldots, C_{m}$ where $\left|C_{i}\right|=k_{i}, i=1,2, \ldots, m$. We can imagine these as being drawn in a plane. The vertices of these cycles are $X=$ $\left\{x_{1}, x_{2}, \ldots, x_{n}\right\}$. We will assume that these cycles have an (arbitrary) orientation. Then for each $x \in X$ there is a predecessor $\nu(x)$ on the same cycle as $x$. As we go we expose a random mapping $f$ from $[n] \rightarrow X$ and then $\pi=f^{-1} \nu f$. If we establish that $f(i)=x_{k}$ and $f(j)=x_{l}$ then we will also establish the length of the edge $\left(x_{k}, x_{l}\right)$ as $\mathbf{A}(i, j)$.

The vertices of $X$ and $[n]$ are divided into exposed and unexposed. $v \in[n]$ is exposed iff $f(v)$ has been determined and $x \in X$ is exposed iff $f^{-1}(x)$ has been determined.

\section{The algorithm}

We break our algorithm into 2 phases: A Greedy Phase and an Extension-Rotation Phase.

\subsection{The Greedy Phase}

We start by deleting an edge of $C_{1}$. This leaves a path $P_{0}$. In general, we have a path $P$, with endpoints $a_{0}, a_{1}$. Initially $P=P_{0}$. We further have a collection of cycles $\mathcal{C}=\mathcal{C}_{S} \cup \mathcal{C}_{L}$ where $\mathcal{C}_{S}=\left\{C \in \mathcal{C}:|C| \leq(\log n)^{2}\right\}$. Initially $\mathcal{C}=C_{2}, C_{3}, \ldots, C_{m}$. We define the set of vertices $R=\bigcup_{C \in \mathcal{C}_{S}} C$. All of the vertices of $P$ will be exposed. Most of $R$ will be unexposed. We end the Greedy Phase when $|R|$ first drops below $\frac{n}{\sqrt{\ln n}}$.

$U_{R}$ denotes the set of unexposed vertices in $R$ and $U_{n}$ denotes the unexposed vertices of $[n]$. We never allow the number of exposed vertices in $R$ to reach more than $10 \frac{n}{\ln n}$. We terminate the algorithm and fail if we expose this number.

A general step of this phase involves the following normal substeps:

(S1) Determine $f^{-1}\left(a_{1}\right)$ by a random choice from $U_{n}$.

(S2) Determine the shortest acceptable (defined below) edge $\left(a_{1}, x\right)$ from $a_{1}$ to a vertex $\xi$ of $\mathcal{C}_{S}$ for which $\nu(\xi)$ is unexposed. Assume that $x$ lies in cycle $C$. Delete the edge $\left(x^{\prime}=\nu(x), x\right)$ from $C$ to create a path $Q$. Now replace $P$ by $P+Q$ and delete $C$ from $\mathcal{C}$. 
Thus each step reduces the number of cycles left by one, at a cost of less than the length of the edge $\left(a_{1}, x\right)$.

In step S2 above, $a_{1}$ is replaced by $x^{\prime}$. So we want $x^{\prime}$ to be unexposed and to have many unexposed vertices which are close to it. Let

$$
\begin{array}{lll}
\omega_{1}=2(\ln n)^{1 / 4} & \omega_{2}=(\ln n)^{1 / 3} & \epsilon=(\ln n)^{-1 / 5} \\
\omega_{1}^{\prime}=2(\ln n)^{5} & \omega_{2}^{\prime}=(\ln n)^{7} & \epsilon^{\prime}=(\ln n)^{-1}
\end{array}
$$

$\xi \in R$ is $\operatorname{good}$ if

(i) $\xi$ is unexposed and

(ii) the $\omega_{1}$ th nearest unexposed vertex in $R$ is $\leq \frac{\omega_{2}}{|R|}$ away and

(iii) the $\omega_{1}^{\prime}$ th nearest unexposed vertex in $R$ is $\leq \frac{\omega_{2}^{\prime}}{|R|}$ away and

(iv) if the cycle containing $v$ is $C$ then either

(a) $|R \backslash C| \leq \frac{n}{\sqrt{\ln n}}$ or

(b) $|R \backslash C|>\frac{n}{\sqrt{\ln n}}$, at most $1 / 2$ of $\xi$ 's first $\omega_{1}$ nearest unexposed vertices in $R$ are in $C$ and at most $1 / 2$ of $\xi$ 's first $\omega_{1}^{\prime}$ nearest unexposed vertices in $R$ are in $C$.

We define the edge $\left(a_{1}, x\right)$ to be acceptable if $x^{\prime}$ is good.

We check now that the search for $x$ can actually be done without exposing too many vertices: We know $\tilde{a}=f^{-1}\left(a_{1}\right)$. We go through $\xi \in f^{-1}(R)$ in increasing order of $\mathbf{A}(\tilde{a}, \xi)$. If $\xi$ is exposed then we go on to the next $\xi$. If $\xi$ is unexposed then we choose $\eta=f(\xi)$ randomly from $U_{R}$. Let $\eta^{\prime}=\nu(\eta)$. If $\eta^{\prime}$ has been exposed, we go onto the next $\xi$. Otherwise we expose $\eta^{\prime}$ by randomly choosing $\zeta=f^{-1}\left(\eta^{\prime}\right)$ from $U_{n}$. We then check to see whether or not $\eta^{\prime}$ is good. We go through $\xi \in f^{-1}(R)$ in increasing order of $\mathbf{A}(\zeta, \xi)$ and we examine the first $\omega_{1}^{\prime}$ unexposed $\xi$ and see whether conditions (ii), (iii) of goodness are satisfied. We do not expose these $\xi$ unless $\eta^{\prime}$ passes this test and we take $x^{\prime}=\eta^{\prime}$.

We need to be sure that in Step S2 we are likely to find a short acceptable choice of edge. Before considering the expected length of the accepted edge, we mention what are the only possibilities: Recall that $C$ is the cycle containing $x$.

(A) $|R \backslash C| \leq \frac{n}{\sqrt{\ln n}}$.

(B) $|R \backslash C|>\frac{n}{\sqrt{\ln n}}$, at most $1 / 2$ of $\xi$ 's first $\omega_{1}$ nearest unexposed vertices in $R$ are in $C$ and at most $1 / 2$ of $\xi$ 's first $\omega_{1}^{\prime}$ nearest unexposed vertices in $R$ are in $C$.

(C) $|R \backslash C|>\frac{n}{\sqrt{\ln n}}$ and more than $1 / 2$ of $\xi$ 's first $\omega_{1}$ nearest unexposed vertices in $R$ are in $C$.

(D) $|R \backslash C|>\frac{n}{\sqrt{\ln n}}$ and more than $1 / 2$ of $\xi$ 's first $\omega_{1}^{\prime}$ nearest unexposed vertices in $R$ are in $C$.

In Case $\mathrm{A}$ we terminate the Greedy Phase and begin the Extension-Rotation phase. Otherwise we can expose the vertices of $C$ and determine which of Cases B-D we are in. Now,

$$
\operatorname{Pr}(C \text { or } D) \leq 2^{\omega_{1}}\left(\frac{(\ln n)^{5 / 2}}{n}\right)^{\omega_{1} / 2}+2^{\omega_{1}^{\prime}}\left(\frac{(\ln n)^{5 / 2}}{n}\right)^{\omega_{1}^{\prime} / 2} \leq n^{-10}
$$


and this probability is small enough that we can afford to fail if either Case $\mathrm{C}$ or D happens. We assume therefore that we do not come across these cases. They would cause trouble, because when we extend $P$ by adding $C$, we would find that many of the close unexposed vertices to $x^{\prime}$ are on the new path and are therefore unusable.

We next need to estimate the expected length of the edge $\left(a_{1}, x\right)$ in Case B.

Lemma 10 Suppose the following holds as $n \rightarrow \infty$,

$$
\epsilon_{0} \rightarrow 0, \quad \frac{\epsilon \omega_{2}}{\ln \ln n} \rightarrow \infty, \quad \frac{\omega_{2}}{\omega_{1} \ln \omega_{2}} \rightarrow \infty
$$

Whp, for every $K \subseteq[n], k=|K| \geq n / \sqrt{\ln n}$ and $L \subseteq K,|L| \leq \epsilon_{0} k$ there are $\leq \epsilon k$ vertices whose $\omega_{1}$ th closest neighbour in $K \backslash L$ is at $\mathbf{M}$ distance $\geq \frac{\omega_{2}}{k}$.

Proof The probability that there exist $K, L$ not satisfying the conditions of the lemma is at most

$$
\begin{aligned}
\left(\begin{array}{c}
n \\
k
\end{array}\right)\left(\begin{array}{c}
k \\
\epsilon k
\end{array}\right)\left(\begin{array}{c}
k \\
\epsilon_{0} k
\end{array}\right)\left(\sum_{i=0}^{\omega_{1}}\left(\begin{array}{c}
k\left(1-\epsilon_{0}\right) \\
i
\end{array}\right)\left(\frac{\omega_{2}}{k}\right)^{i}\left(1-\frac{\omega_{2}}{k}\right)^{k\left(1-\epsilon_{0}\right)-i}\right)^{\epsilon k} \\
\leq\left(\frac{n e}{k} \cdot\left(\frac{e}{\epsilon}\right)^{\epsilon} \cdot\left(\frac{e}{\epsilon_{0}}\right)^{\epsilon_{0}}\left(\left(\left(1-\epsilon_{0}\right) \omega_{2}\right)^{\omega_{1}} e^{-\omega_{2}\left(1-\epsilon_{0}\right)}\right)^{\epsilon}\right)^{k} \rightarrow 0 .
\end{aligned}
$$

We know that $a_{1}$ was either obtained from $C_{1}$ or was a good vertex chosen in the previous step. In both cases there were at least $\omega_{1}$ (resp. $\left.\omega_{1}^{\prime}\right)$ edges of length $\leq \frac{\omega_{2}}{|R|}\left(\right.$ resp. $\left.\leq \frac{\omega_{2}^{\prime}}{|R|}\right)$ to unexposed members of $R$. Because cases $\mathrm{C}, \mathrm{D}$ are ruled out, at most $1 / 2$ of these are absorbed into $P$. Using Lemma 10 with $\epsilon_{0}=\frac{10}{\sqrt{\ln n}}$ (a bound on the proportion of exposed vertices in $R$ ) we see that the expected length of the edge $\left(a_{1}, x\right)$ is at most

$$
\frac{\omega_{2}}{|R|}+\left(\epsilon_{0}+\epsilon+\epsilon^{\prime}\right)^{\omega_{1} / 2} \frac{\omega_{2}^{\prime}}{|R|}+\left(\epsilon_{0}+\epsilon+\epsilon^{\prime}\right)^{\omega_{1}^{\prime} / 2} \leq \frac{2(\ln n)^{1 / 3}}{|R|} .
$$

Explanation Assume the condition of Lemma 10 holds. Let $K=R$ and $L=R \backslash U_{R}$. $\epsilon_{0}+\epsilon+\epsilon^{\prime}$ bounds the probability that for a randomly chosen vertex $x$ of $R$ has $\nu(x) \notin U_{R}$ or $\nu(x)$ fails tests (ii), (iii) of goodness.

Furthermore,

$\mathbf{E}$ (number of vertices exposed per step before finding an acceptable edge $\mid$ previous steps)

$$
\leq \frac{1}{1-\left(\epsilon_{0}+\epsilon+\epsilon^{\prime}\right)} \leq 2
$$

It is not therefore difficult to show that whp we never expose more than $\frac{9 n}{\ln n}$ vertices due to these steps.

We now return to the main cost of the Greedy Phase (as defined in (6)).

We remind the reader that the lengths of the cycles in the optimal 2-factor $F^{*}$ are $k_{1}, k_{2}, \ldots, k_{m}$ where $m \leq 3 \frac{n}{\ln n}$ and $k_{1}=\min _{i} k_{i}$.

We can take $k_{1} \leq(\ln n)^{2}$ for otherwise $m \leq n /(\ln n)^{2}$ and we can dispense with a Greedy Phase and just use the Extension-Rotation Phase. In this Phase whp we remove a cycle at the cost of 
$O\left(\frac{\ln n}{n}\right)$ per cycle. Thus in the case of $k_{1} \geq(\ln n)^{2}$ we can whp find a tour of length $O\left((\ln n)^{-1}\right)$ more than the length of $F^{*}$.

We now wish to bound the expected sum of the lengths of the edges added in normal steps. Suppose that we have re-ordered the cycles so that they are absorbed into $P$ in order $1,2, \ldots$. Assume that $\mathcal{C}_{L}=\left\{C_{\rho+1}, C_{\rho+2}, \ldots, C_{m}\right\}$ and let $K_{L}=\sum_{i=\rho+1}^{m} k_{i}$.

Let $L_{i}=n-k_{1}-k_{2}-\cdots-k_{i}-K_{L}=k_{i+1}+k_{i+2}+\cdots+k_{\rho}, m^{\prime}=\min \left\{i: L_{i} \leq \frac{n}{\sqrt{\ln n}}\right\}$. We note that the Greedy Phase is only concerned with the first $m^{\prime}-1$ cycles. Next let $I=\left\{i: k_{i} \leq\right.$ $\left.L=\frac{\ln n}{2 \ln \ln n}\right\}$. We can assume (Lemma 9) that $|I| \leq n^{3 / 4}$. Then let

$$
S\left(m^{\prime}\right)=\sum_{i=1}^{m^{\prime}-1} \frac{(\ln n)^{1 / 3}}{L_{i}} .
$$

It will suffice to show that $S\left(m^{\prime}\right)=o(1)$. For then (6) will imply that the expected weight of the edges added in the Greedy Phase is $o(1)$ and so is $o(1)$ whp by the Markov inequality.

$$
\begin{aligned}
\sum_{i=1}^{m^{\prime}-1} \frac{1}{L_{i}} & \leq|I| \frac{\sqrt{\ln n}}{n}+\sum_{i=0}^{m^{\prime}-1} \frac{1}{L_{1}-i L} \\
& \leq O\left(n^{-1 / 4} \sqrt{\ln n}\right)+\int_{x=0}^{m^{\prime}} \frac{d x}{L_{1}-x L} \\
& =O\left(n^{-1 / 4} \sqrt{\ln n}\right)+\frac{1}{L} \ln \left(\frac{L_{1}}{L_{1}-m^{\prime} L}\right) \\
& =O\left((\ln n)^{-1 / 2}\right)
\end{aligned}
$$

since $L_{1}-m^{\prime} L \geq L_{m^{\prime}-1}-(|I|+1) L \geq \frac{n}{2 \sqrt{\ln n}}$ and $m^{\prime} \leq \frac{3 n}{\ln n}$.

Thus $S\left(m^{\prime}\right)=O\left((\ln n)^{-1 / 6}\right)$ and we are done.

\subsection{Final Extension-Rotation Phase}

We enter this phase with a path $P$ and, most importantly, only $m^{\prime \prime}=m-m^{\prime}+\left|\mathcal{C}_{L}\right|=O\left(\frac{n \ln \ln n}{(\ln n)^{3 / 2}}\right)$ cycles. We will absorb each cycle into $P$ at an expected cost of $O\left(\frac{\ln n}{n}\right)$ and with the same cost turn the final $n-1$ edge Hamilton path into a tour and so complete the proof of Theorem 1.

We will use rotations and $\Gamma_{20000}$ of Section 2.3 for this task. At a general stage we have, as usual, a path $P$ plus a collection of vertex disjoint cycles $\mathcal{C}$ which cover a set of vertices $R$. Let the endpoints of $P$ be $a, b$.

If $a$ (or $b$ ) has a $\Gamma_{20000}$-neighbour $x$ in $R$ then we replace $P$ by $P+C-\left(x, x^{\prime}\right)$ where $C$ is the cycle containing $C$ and $x^{\prime}$ is a neighbour of $x$ on $C$. We do not need to be concerned anymore with good or bad vertices. We call this operation, extending $P$ and for every path obtained by rotation, we also see if an extension is possible. So, in the discussion of rotations below, assume that no extension is possible for any path produced.

For a path $P=a=a_{0} a_{1}, \ldots, a_{h}$ and edge $a_{h} a_{i}$ we say that the path $P^{\prime}=a_{0} a_{1} \ldots a_{i} a_{h} a_{h-1} \ldots a_{i+1}$ is obtained from $P$ by a rotatoin with $a_{0}$ as fixed endpoint. For a vertex $v \in P$ let $\rho(v)$ be the minimum number of rotations, with $a$ fixed, needed to construct a path with $v$ as an endpoint. $\rho(v)=\infty$ if it is not possible to construct such a path. Then let

$$
S(P, a, t)=\{v \in P: \rho(v)=t\} .
$$


It follows from Lemma 3 that

$$
|S(P, a, t)| \leq n / 25 \text { implies }|S(P, a, t+1)| \geq 9|S(P, a, t)|
$$

Indeed, assuming no extensions are possible, and proceeding inductively,

$$
|S(P, a, t+1)| \geq \frac{1}{2}\left(\left|N_{20000}(S(P, a, t))\right|-\sum_{\tau=0}^{t}|S(P, a, \tau)|\right) \geq \frac{1}{2}\left(20-\frac{9}{8}\right)|S(P, a, t)| .
$$

It follows that for some $t^{*} \leq \log _{9} n / 25$ we find that $\left|S\left(P, a, t^{*}+1\right)\right| \geq 9 n / 25$. Let $E N D(a)=$ $S\left(P, a, t^{*}+1\right)$.

Now we have for each $v \in E N D(a)$ a path $P_{v}$ with endpoints $a, v$ which goes through all vertices of $P$ (unless we have found an extension). For each such $v$ construct the set $E N D(v)=$ $S\left(P_{v}, v, t_{v}\right), t_{v} \leq 1+\log _{9} n / 25$ for which $|E N D(v)| \geq 9 n / 25$. Putting $E N D=\{a\} \cup E N D(a)$ we see that we have created a collection of sets $E N D(v), v \in E N D$, each of size $\geq 9 n / 25$ with the property that

$v \in E N D, w \in E N D(v)$ implies that there is a path $P[v, w]$ with endpoints $v, w$ going

through all vertices of $P$ and such that $P[v, w]$ differs from $P$ in at most

$2\left(1+\log _{9} n / 25\right)$ edges

Now we can use the Red copy of $G_{n, p_{0}}$ (see Section 2.4) to find a Red edge joining some $v \in E N D$ to $w \in E N D(v)$. Whp we only need to check $O(\ln n) \operatorname{such}$ pairs $v, w$ for each cycle before finding a red edge, $O(n)$ pairs altogether. thus we will whp find a Red edge each time we need to.

Once we have turned $P$ into a cycle $C$, we can use the fact that whp $\Gamma_{s}$ is connected for $s \geq 2$, Fenner and Frieze [5], to assert the existence of a $\Gamma_{20000}$ edge joining $C$ to $\mathcal{C}$. In the event that $\mathcal{C}$ is empty, we have finished.

The cost of the added Red edges is $O\left(m^{\prime \prime} p_{0}\right)=o(1)$. We can use (2) and (9) to see that whp the total weight of $\Gamma_{20000}$ edges used in this phase is $O\left(m^{\prime \prime} \frac{\ln n}{n}\right)=o(1)$ and we are finished with the proof of Theorem 1.

As a final remark we observe that in order to improve and simplify the above analysis, it would be most fruitful to try to improve the result of Lemma 2.

\section{References}

[1] D. Aldous, The zeta(2) Limit in the Random Assignment Problem. Random Structures and Algorithms 18 (2001) 381-418.

[2] E. Balas and P. Toth, Branch and bound methods, in The traveling salesman problem: a guided tour of combinatorial optimization, E.L.Lawler, J.K.Lenstra, A.H.G.Rinnooy Kan and D.B.Shmoys Eds., Wiley, 1985.

[3] C.Cooper and A.M.Frieze, Hamilton cycles in random graphs and digraphs, Random Structures and Algorithms 16 (2000) 369-401.

[4] M.E. Dyer and A.M. Frieze, On patching algorithms for random asymmetric travelling salesman problems, Mathematical Programming 46 (1990) 361-378.

[5] T.I. Fenner and A.M.Frieze, On the connectivity of random $m$-orientable graphs and digraphs, Combinatorica 2, 347-359 
[6] A.M. Frieze, On the value of a random minimum spanning tree problem, Discrete Applied Mathematics 10 (1985) 47-56.

[7] A.M. Frieze and G.R. Grimmett, The shortest path problem for graphs with random arclengths, Discrete Applied Mathematics 10 (1985) 57-77.

[8] A.M. Frieze and G. Sorkin, The probabilistic relationship between the assignment and asymmetric traveling salesman problems, Proceedings of SODA 2001, 652-660

[9] R.M. Karp, A patching algorithm for the non-symmetric traveling salesman problem, SIAM Journal on Computing 8 (1979) 561-573.

[10] R.M. Karp and J.M. Steele, Probabilistic analysis of heuristics, in The traveling salesman problem: a guided tour of combinatorial optimization, E.L.Lawler, J.K.Lenstra, A.H.G.Rinnooy Kan and D.B.Shmoys Eds., Wiley, 1985.

[11] L. Lovász and M.D. Plummer, Matching Theory, North Holland, 1986.

[12] D.W. Walkup, On the expected value of a random asignment problem, - em SIAM Journal on Computing 8 (1979) 440-442.

This research was sponsored in part by National Science Foundation (NSF) grant no. CCR-0122581. 